\title{
Soft X-Ray and Extreme Ultraviolet Excess Emission from Clusters of Galaxies
}

\author{
F. Durret · J.S. Kaastra $\cdot$ J. Nevalainen $\cdot$ T. Ohashi • \\ N. Werner
}

Received: 17 September 2007 / Accepted: 22 October 2007 / Published online: 15 February 2008 (C) Springer Science+Business Media B.V. 2008

\begin{abstract}
An excess over the extrapolation to the extreme ultraviolet and soft X-ray ranges of the thermal emission from the hot intracluster medium has been detected in a number of clusters of galaxies. We briefly present each of the satellites (EUVE, ROSAT PSPC and BeppoSAX, and presently XMM-Newton, Chandra and Suzaku) and their corresponding instrumental issues, which are responsible for the fact that this soft excess remains controversial in a number of cases. We then review the evidence for this soft X-ray excess and discuss the possible mechanisms (thermal and non-thermal) which could be responsible for this emission.
\end{abstract}

Keywords Galaxies: clusters $\cdot$ X-ray: spectra

\footnotetext{
F. Durret $(\bowtie)$

Institut d'Astrophysique de Paris, CNRS, UMR 7095, Université Pierre et Marie Curie, 98bis Bd Arago, 75014 Paris, France

e-mail: durret@iap.fr

J.S. Kaastra $\cdot$ N. Werner

SRON Netherlands Institute for Space Research, Sorbonnelaan 2, 3584 CA Utrecht, The Netherlands

J.S. Kaastra

Astronomical Institute, Utrecht University, P.O. Box 80000, 3508 TA Utrecht, The Netherlands

J. Nevalainen

Observatory, University of Helsinki, P.O. Box 14, 00014 Helsinki, Finland

T. Ohashi

Department of Physics, Tokyo Metropolitan University, 1-1 Minami-Osawa, Tokyo 192-0397, Japan

N. Werner

Max-Planck-Institute für Astrophysik, Karl-Schwarzschild-Strasse 1, 85749, Garching, Germany
} 


\section{Introduction}

The existence of soft excess emission originating from clusters of galaxies, defined as emission detected below $1 \mathrm{keV}$ in excess over the usual thermal emission from hot intracluster gas (hereafter the ICM) has been claimed since 1996. Soft excesses are particularly important to detect because they may (at least partly) be due to thermal emission from the Warm-Hot Intergalactic Medium, where as much as half of the baryons of the Universe could be. They are therefore of fundamental cosmological importance.

Soft excess emission has been observed (and has also given rise to controversy) in a number of clusters, mainly raising the following questions: (1) Do clusters really show a soft excess? (2) If so, from what spatial region(s) of the cluster does the soft excess originate? (3) Is this excess emission thermal, originating from warm-hot intergalactic gas (at temperatures of $\sim 10^{6} \mathrm{~K}$ ), or non-thermal, in which case several emission mechanisms have been proposed. Interestingly, some of the non-thermal mechanisms suggested to account for soft excess emission can also explain the hard X-ray emission detected in some clusters, for example by RXTE and BeppoSAX (also see Petrosian et al. 2008-Chap. 10, this issue; Rephaeli et al. 2008-Chap. 5, this issue).

Several instruments have been used to search for soft excess emission: EUVE, ROSAT and BeppoSAX in the 1990s, and presently XMM-Newton, Chandra and Suzaku. We will briefly present a history of these detections, emphasising the difficulties to extract such weak signal and the underlying hypotheses, and summarising what is known on each of the observed clusters. For clarity, results will be presented separately for the various satellites. Finally we summarise and discuss the various models proposed to account for Extreme U1traviolet (hereafter EUV) emission with their pros and cons.

\section{Instrumental Issues}

\subsection{The Extreme Ultraviolet Explorer (EUVE) Satellite}

The EUVE satellite was launched in 1992 and operated till 2001, covering the 50-250 eV energy range (Bowyer and Malina 1991). The rectangular shape of the Lex/B (65-248 eV) filter resulted in images where the length of one side of the image greatly exceeds the other side: $\sim 40^{\prime}$ in width and more than $2^{\circ}$ in length (see e.g. Fig. 1 in Durret et al. 2002).

The spatial scale was 13 pixels/arcmin. Due to the limited sensitivity of EUVE, exposure times on clusters were typically between several tens of ks and 1 Ms.

\subsection{ROSAT PSPC}

The Position Sensitive Proportional Counter PSPC instrument on board the ROSAT satellite first detected the soft excess component of clusters of galaxies at X-ray wavelengths. The PSPC had an effective area of about $200 \mathrm{~cm}^{2}$ at $0.28 \mathrm{keV}$, enabling soft X-ray studies. It had a large field of view (hereafter FOV) of 50' radius, covering the virial radius in most clusters and enabling in most cases the estimation of the local background. The low and stable internal background of the PSPC allowed reliable X-ray measurements at large radii where the background is important. A major problem for soft excess studies with the PSPC is that it did not cover energies above $2 \mathrm{keV}$. Thus, it could not be used to determine reliably the hot gas properties, which had to be measured elsewhere. A further limitation was the low energy resolution which did not allow detection of possible emission line blends emanating from the soft component. The angular resolution of the ROSAT PSPC was $\sim 15^{\prime \prime}$. Details on the ROSAT PSPC instrument can be found in Briel et al. (1996). 


\subsection{XMM-Newton EPIC}

An important change in the study of the soft excess came with the XMM-Newton satellite. The XMM-Newton European Photon Imaging Camera (EPIC) instruments PN and MOS extend the energy band coverage to $10 \mathrm{keV}$, thus enabling simultaneous determination of the hot gas and soft excess component properties.

The large collecting area of the EPIC telescopes $\left(\sim 1000 \mathrm{~cm}^{2}\right.$ at $0.5 \mathrm{keV}$ for the PN) provides the high statistical quality data necessary to examine the few $10 \%$ soft excess effect on top of the hot gas emission. The spectral resolution of the PN at $0.5 \mathrm{keV}$ is $60 \mathrm{eV}$ (FWHM), rendering it possible to resolve the emission lines emanating from the soft excess component. However, the relatively small FOV ( $15^{\prime}$ radius $)$ of EPIC prevents the study of the cluster outskirts for the nearest clusters with single pointings. The usage of offset pointings introduce the complex stray light problem which complicates the analysis of weak signals such as the soft excess. The more distant clusters, which would be covered out to the virial radius with a single pointing, are fainter, which reduces the quality of the signal. Thus, the XMM-Newton soft excess analysis is mostly limited to the central regions of nearby clusters. Details on the characteristics of XMM-Newton can be found in Turner et al. (2001) and Strüder and Briel (2001), or in Ehle et al. (2006).

A further complication is the strong and flaring particle-induced detector background. A local background estimate is vital when analysing weak signals such as the cluster soft excess. This is usually not available for nearby clusters since they fill the FOV, and one has to resort to blank-sky based background estimates. This introduces uncertainties in the analysis, and further limits the cluster analysis to central regions where the background is not important. Together with the FOV limitations, the background problem limits the usefulness of XMM-Newton for measuring the soft excess in a large cluster sample.

The on-going calibration work on the PN and MOS instruments resulted in changes in the derived soft excess properties for a few clusters (Nevalainen et al. 2007). Thus, the EPIC results have some degree of systematic uncertainty based on calibration inaccuracies, and definitive results on the soft excess properties are not yet available (also see Sect. 4.1.2).

\subsection{Chandra}

Chandra, launched in the same year as XMM-Newton (1999), has a similar eccentric orbit as the latter satellite and therefore suffers from comparable enhanced background problems. Its angular resolution is much higher ( $0.8^{\prime \prime}$ Half Energy Width) than that of XMMNewton $\left(14^{\prime \prime}\right)$. The advantage is that for extended sources like clusters of galaxies subtraction of contaminating background point sources can be done more accurately, and more importantly, blurring effects by the point spread function of the telescope can usually be ignored. These blurring effects were a serious problem for the BeppoSAX LECS data (Sect. 3.2.2). However, the effective area at low energies $(E<0.5 \mathrm{keV})$ of Chandra is an order of magnitude smaller than that of XMM-Newton, and time-dependent contamination that affects in particular the lower energies is a complicating factor in the analysis of Chandra data.

\subsection{Suzaku XIS}

Suzaku is the fifth Japanese X-ray astronomy satellite, launched in July 2005 (Mitsuda et al. 2007). Unfortunately, the main observing instrument XRS, consisting of X-ray microcalorimeters used in space for the first time, did not last until the first space observations, 
due to the loss of liquid He (Kelley et al. 2007). On the other hand, the XIS instrument, which employs X-ray CCDs with improved performance for X-ray spectroscopy, is functioning well (Koyama et al. 2007). Regarding soft X-ray spectral studies of diffuse sources, XIS offers the best capability so far achieved with X-ray satellites.

The XIS system consists of 4 units of mirror and detector combinations. The X-ray mirrors are identical and have a focal length of $4.5 \mathrm{~m}$ and an effective area of about $500 \mathrm{~cm}^{2}$ at $2 \mathrm{keV}$ (Serlemitsos et al. 2007). The angular resolution is about $2^{\prime}$, limited by the lightweight design of the thin foil mirror.

The 4 CCDs in the focal plane operate jointly during the observations. The chips are square with an area of $10 \mathrm{~mm} \times 10 \mathrm{~mm}$ and the number of pixels is $10^{6}$. The field of view is $17^{\prime} \times 17^{\prime}$. One chip is back-illuminated, which gives a superior soft $\mathrm{X}$-ray sensitivity with somewhat poorer energy resolution and higher background above $7 \mathrm{keV}$. The other 3 chips are of standard front illumination type. The typical energy resolution was $150 \mathrm{eV}$ FWHM at $5.9 \mathrm{keV}$ at the time of launch. The resolution degraded significantly with time ( $200 \mathrm{eV}$ after 1 year), and the XIS team performed a charge injection operation after October 2006 to maintain the resolution around $170 \mathrm{eV}$. In November 2006, one of the $3 \mathrm{FI}$ (front illumination) chips developed excess noise, and it has been switched off since then. This leaves a total of 3 chips, one BI (back illumination) and 2 FIs, in operation. Its background is lower than that of XMM-Newton.

\section{Soft X-Ray Excess Emission Based on EUVE and ROSAT PSPC Data}

\subsection{The First Objects with a Soft X-Ray Excess Discovered: Virgo (Redshift $z=0.0038$ ) and Coma $(z=0.0231)$}

The first mention of soft excess emission in the $0.065-0.245 \mathrm{keV}$ band was made by Lieu et al. (1996a) for the Virgo cluster, based on data obtained with the EUVE satellite. These authors detected emission around M 87 up to a radial distance of $\sim 20^{\prime}$, and found excess emission over the best fit single temperature plasma model obtained by fitting simultaneously the EUVE and ROSAT PSPC 0.18-2.0 keV data. This excess was found to decrease with radial distance (see Fig. 1). Similar results were then found for the Coma cluster, also based on EUVE and ROSAT data, by Lieu et al. (1996b).

The first interpretation proposed was that the soft excess emission was thermal, with a single component of ICM gas at a temperature between $5 \times 10^{5} \mathrm{~K}$ and $10^{6} \mathrm{~K}$ for Virgo (Lieu et al. 1996a) and two components at $8 \times 10^{5} \mathrm{~K}$ and $2 \times 10^{6} \mathrm{~K}$ for Coma (Lieu et al. 1996b). The immediate implication of this hypothesis was that such warm gas would cool very rapidly, implying a very high mass accretion rate of several hundred Solar masses per year, and therefore the production of a large amount of gas in the cool phase (of the order of $10^{14} \mathrm{M}_{\odot}$, Lieu et al. 1996a, 1996b).

A second model was then proposed by Sarazin and Lieu (1998), in which soft excess emission was due to the Inverse Compton emission (hereafter IC) of cosmic microwave background photons on a relic population of cosmic ray electrons. Such electrons could have been produced by supernovae, by radio galaxies or by particle acceleration in intracluster shocks. Their energy would amount to $1-10 \%$ of the thermal energy content of the ICM. The inverse Compton model was also favoured by Bowyer and Berghöfer (1998), and Lieu et al. (1999a) suggested that cosmic rays could be as energetically important as the thermal ICM. 

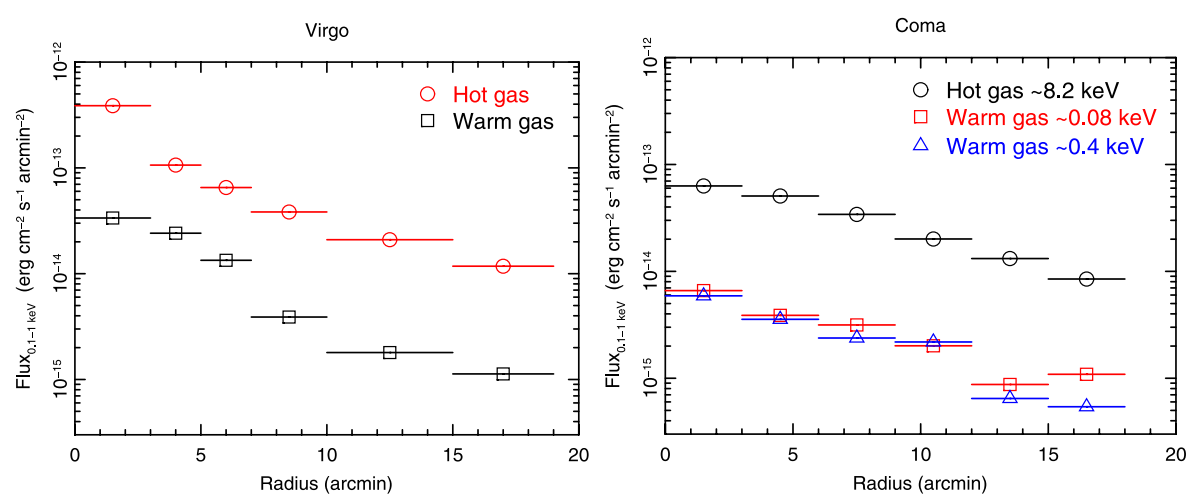

Fig. 1 Left panel: Radial profiles of the $0.1-1.0 \mathrm{keV}$ surface brightness of the hot ICM and of the warm gas in Virgo found by Lieu et al. (1996a). Right panel: Radial profile of the $0.1-1.0 \mathrm{keV}$ surface brightness of the hot ICM and of the two warm gas components in Coma found by Lieu et al. (1996b)

From ROSAT images at low energy, Bonamente et al. (2001a) derived the existence of large amounts of cold gas in the ICM of Virgo and Coma, confirming the existence of a multiphase ICM.

More clusters were then observed, Virgo and Coma were reobserved, and controversy began to arise concerning the very existence of a soft excess over the thermal emission from hot gas responsible for cluster X-ray emission.

An agreement on the existence of soft excess emission based on EUVE and ROSAT PSPC data was reached by different observers only on the Virgo and Coma clusters. By taking into account the variations of telescope sensitivity over the field of view, Bowyer et al. (1999) confirmed the presence of EUV excess emission in Coma. These authors also later confirmed the soft excess in Virgo and interpreted it as due to inverse Compton radiation (Berghöfer et al. 2000a). With EUVE in situ background measurements, Bonamente et al. (2001b) agreed with the existence of an EUV excess in Virgo and found that this excess increased with radius.

Using PSPC data, Bonamente et al. (2003) found a very large scale (2.6 Mpc) soft excess halo in the Coma cluster, exceeding the thermal emission by $200 \%$ at the largest radii. Note that at a distance of $40^{\prime}$ the soft excess flux exceeds that of the hot gas by $100 \%$, consistent with the later XMM-Newton analysis of Finoguenov et al. (2003) at the same distance. The non-thermal model gave a poor fit to the spectrum of the soft excess, while the thermal model was acceptable, with a best-fit temperature of $\sim 0.2 \mathrm{keV}$.

\subsection{Other Clusters Observed with EUVE and ROSAT}

\subsubsection{Abell $1795(z=0.0625)$}

A strong soft excess was found in Abell 1795 from EUVE data by Mittaz et al. (1998). Later, Bonamente et al. (2001b) confirmed the existence of this excess by observing with EUVE an in situ background, and found that, as for Virgo, the EUV excess increased with radius.

However, these results were debated: by taking into account the variations of telescope sensitivity over the field of view, Bowyer et al. (1999) claimed that there was no evidence for EUV excess in Abell 1795 and later confirmed this result from BeppoSAX data (Berghöfer and Bowyer 2002). However, the existence of soft excess emission in this cluster was confirmed by Durret et al. (2002) from EUVE and ROSAT PSPC data. Soft excess in Abell 1795 
was also found with the ROSAT PSPC (Bonamente et al. 2003) at the 10-20\% level in the $0.2-0.4 \mathrm{keV}$ band, and by Nevalainen et al. (2003) and Kaastra et al. (2003), both based on XMM-Newton data (see Sects. 4.2.1 and 4.2.2, respectively).

\subsubsection{Abell $2199(z=0.0301)$}

The presence of a soft excess was disputed in Abell 2199. From EUVE data, Lieu et al. (1999b) detected a soft excess, and confirmed its existence up to a radius of 20' using a background measured from offset pointing Lieu et al. (1999c). Confirmation of the existence of a soft and a hard excess in this cluster was given by Kaastra et al. (1999) based on BeppoSAX, EUVE and ROSAT data. A three phase ICM was proposed to account for the observations by Lieu et al. (2000).

On the other hand, from other EUVE observations taking into account the variations of telescope sensitivity over the field of view, Bowyer et al. (1999) found no evidence for EUV excess. They confirmed this result with BeppoSAX data (Berghöfer and Bowyer 2002), but Kaastra et al. (2002) claimed that their analysis was wrong. According to the latter authors, the problem was that Berghöfer \& Bowyer based their conclusion on a plot of the ratio of the observed radial intensity profiles in the $0.5-2.2 \mathrm{keV}$ band as compared to the $0.1-$ $0.3 \mathrm{keV}$ band. Because the point spread function of the BeppoSAX LECS instrument is a strong function of energy ( $10^{\prime}$ FWHM at $E=0.28 \mathrm{keV}$, scaling as $\left.E^{-0.5}\right)$, and the lowenergy counts in the $0.1-0.3 \mathrm{keV}$ band contain a significant contribution from higher energy photons, even for a fully isothermal cluster the ratio of the radial intensities in both bands shows strong $(\sim 50 \%)$ excursions. Moreover, the predicted ratio depends strongly on issues like abundance gradients, modelling of the cooling flow, etc. that were ignored by Berghöfer and Bowyer (2002).

The existence of a soft excess was independently confirmed by Durret et al. (2002) from EUVE and ROSAT PSPC data. The ROSAT PSPC analysis of Bonamente et al. (2003) also revealed marginal evidence for soft excess in Abell 2199, and they derived that the temperature of the soft excess component must be below $0.2 \mathrm{keV}$. Kaastra et al. (2003) confirmed the presence of a soft excess in this cluster from XMM-Newton data.

\subsubsection{Abell $4059(z=0.0460)$}

No soft excess was detected in Abell 4059 by Berghöfer et al. (2000b); strangely, these authors even found a deficit of EUV emission in the central 2 '. However, a soft excess was detected in this cluster by Durret et al. (2002) as described in the next subsection.

\subsubsection{Complementary Results on the Five above Clusters}

A different approach was proposed by Durret et al. (2002) based on the wavelet analysis and reconstruction of EUVE and ROSAT PSPC observations for the five previously discussed clusters: Virgo, Coma, Abell 1795, Abell 2199 and Abell 4059. A soft excess was found in all five clusters, even when taking into account temperature and abundance gradients of the ICM. The radial profiles of the EUV to X-ray ratios are shown in Fig. 2. The EUV and X-ray profiles were shown to differ statistically, suggesting that the EUV and X-ray emissions were probably not due to the same physical mechanism.

\subsubsection{Fornax $(z=0.0046)$}

No soft excess was detected in Fornax by Bowyer et al. (2001) at any scale, but Bonamente et al. (2002) did detect a soft X-ray excess in this cluster, which is part of the sample described in Sect. 3.2.9. 

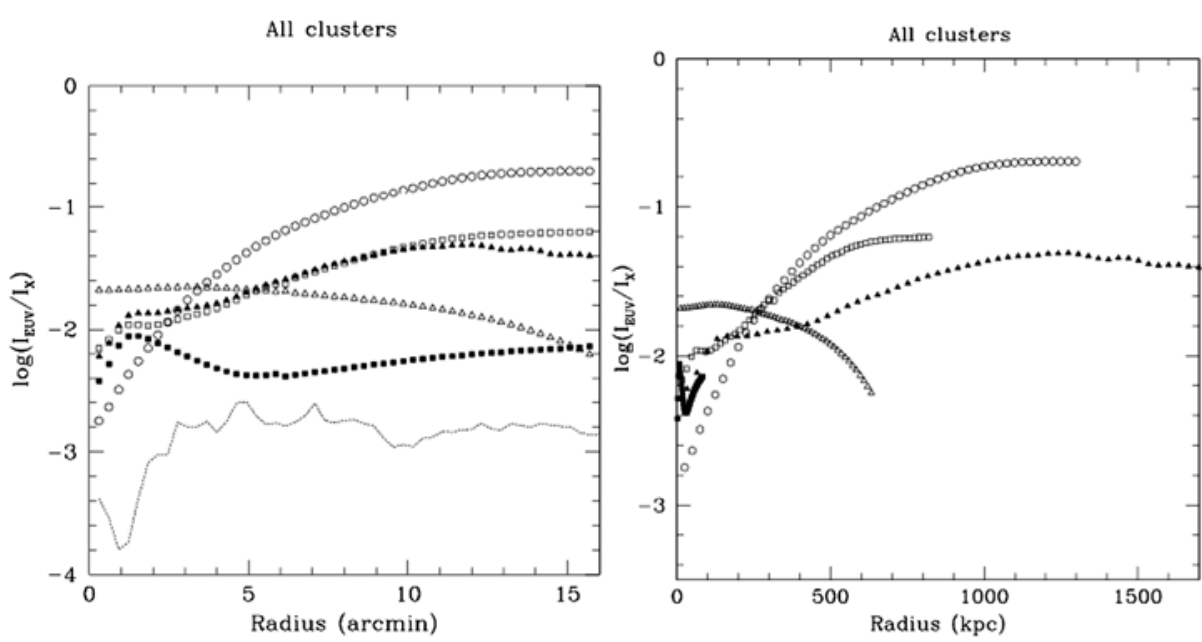

Fig. 2 EUV to X-ray ratio as a function of cluster radius (expressed in arcmin in the left plot and in kpc in the right plot) for the five clusters observed by Durret et al. (2002) with the EUVE and ROSAT PSPC satellites. The symbols are the following: Abell 1795: filled triangles, Abell 2199: empty squares, Abell 4059: empty circles, Coma: empty triangles, Virgo: filled squares. The dashed line in the left figure shows the ratio of the EUVE to ROSAT PSPC PSFs. Error bars are omitted for clarity; they are typically smaller than \pm 0.01 in logarithmic scale, i.e. too small to be clearly visible on the figure

\subsubsection{Shapley Supercluster $(z=0.046)$}

The ROSAT PSPC data of four clusters Abell 3571, Abell 3558, Abell 3560 and Abell 3562 in the Shapley supercluster were analysed by Bonamente et al. (2001c). They modelled the hot gas spectra using published ASCA results (Abell 3571 and Abell 3558) or fitting the PSPC data (Abell 3560 and Abell 3562). These models fit the PSPC data poorly due to excess residuals in the $\mathrm{R} 2$ band $(0.15-0.3 \mathrm{keV})$ in all clusters. Based on the residuals, the authors determined the fractional soft excess emission above the hot gas emission model, amounting to 50\% at most. For Abell 3571 and Abell 3558, they found that the fractional soft excess increased with the cluster radius, an effect already found in the EUVE data of Virgo, Abell 1795, and Abell 2199 (Bonamente et al. 2001b; Lieu et al. 1999b).

BeppoSAX LECS data on Abell 3571 and Abell 3562 supported the PSPC results. The analysis showed that a simple one-temperature thin-plasma model is not adequate to describe the emission.

The data indicated a thermal model with $\mathrm{k} T=0.1 \mathrm{keV}$ for Abell 3558, but the quality of the data was not appropriate for distinguishing the nature of the soft component or modelling its properties in detail.

\subsubsection{Sérsic 159-03 $(z=0.0580)$}

Sérsic 159-03 (also known as AS 1101) was found to show strong soft excess and even to be the brightest soft excess cluster by Bonamente et al. (2001d). These authors combined three PSPC pointings of Sérsic 159-03 obtaining $19 \mathrm{ks}$ of exposure time. The low temperature $(\sim 2 \mathrm{keV})$ of the hot gas allowed its spectroscopic analysis using the PSPC data alone. Extrapolating the best-fit spectral model from the $0.5-2.0 \mathrm{keV}$ band to lower energies revealed a very strong soft excess at $0.2 \mathrm{keV}, 100 \%$ over the hot gas emission. This excess was then confirmed with XMM-Newton (see Sect. 4.1.3). 
Bonamente et al. (2001d) tested the hypothesis that the soft excess could be an artefact caused by a sub-Galactic absorbing column density $N_{\mathrm{H}}$ towards the cluster. However, this yielded an unrealistically low $N_{\mathrm{H}}\left(5 \times 10^{19} \mathrm{~cm}^{-2}\right)$, even lower than that of the global minimum of the Lockman Hole, implying that $N_{\mathrm{H}}$ variation is not the cause of the soft excess in this cluster.

Despite the high quality of the signal, the nature of the soft excess component could not be determined, since non-thermal and thermal models yield statistically equally acceptable fits.

\subsubsection{The Hercules Supercluster $(z \sim 0.035)$}

A soft excess was also detected from low energy ROSAT data in the clusters Abell 2052 $(z=0.0348)$ and Abell $2063(z=0.0348)$ of the Hercules supercluster (Bonamente et al. 2005b); however these authors underlined the difficulty of background subtraction.

\subsubsection{The Bonamente et al. (2002) Sample}

Bonamente et al. (2002) published a ROSAT PSPC analysis of the largest soft excess sample of galaxy clusters so far, 38 in number. They modelled the ROSAT PSPC data of the hot gas using published results from ASCA and BeppoSAX which are more suitable due to their energy coverage up to $10 \mathrm{keV}$. These models were extrapolated to the PSPC channels and compared with the data in the $0.2-0.4 \mathrm{keV}$ band. The analysis yielded significant $(3 \sigma)$ soft excess in $\sim 30 \%$ of the clusters in the sample. The actual fraction of clusters with soft excess may be higher, since the clusters with the deepest ROSAT observations all had soft excess. Using a thermal model with a temperature of $0.08 \mathrm{keV}$ and metal abundance of 0.3 Solar, or a power-law $I=I_{0} E^{-\alpha_{\mathrm{ph}}}$ (where $I$ is the intensity and $E$ the photon energy) of index $\alpha_{\mathrm{ph}}=1.75$, the soft excess fluxes correspond to $0.2-0.4 \mathrm{keV}$ luminosities of $10^{40}-10^{43} \mathrm{erg} \mathrm{s}^{-1}$. The excess was more prominent in the regions outside the central 150-200 kpc.

The indicated increase of the relative strength of the soft excess with radius is qualitatively consistent with the non-thermal Inverse Compton radiation of CMB photons from the relativistic cluster electrons. The hot gas emission is proportional to $n_{\mathrm{e} \text {,thermal }}^{2}$ while the IC is proportional to $n_{\mathrm{e}, \text { relativistic }}$ and the usual assumption is that the relativistic electrons follow the gas distribution. This model however faces energetic problems.

The authors also examined the possibility that the soft excess originates from a large number of unresolved X-ray-emitting cluster galaxies. The most relevant sources of X-rays are Low Mass X-ray Binaries (LMXB) and especially their $\mathrm{k} T \sim 0.25 \mathrm{keV}$ blackbody component coming from the accretion disk. The typical soft X-ray luminosity of a galaxy due to LMXB is of order $10^{38} \mathrm{erg} \mathrm{s}^{-1}$ and thus $10^{3}-10^{4}$ galaxies are needed to produce the typical observed soft X-ray luminosity, while typical numbers of galaxies in rich clusters are of order several hundreds. Thus, unresolved sources in cluster galaxies cannot explain the soft excess. A similar conclusion can be reached by looking at the galaxy X-ray luminosity function, such as that obtained for the Coma cluster by Finoguenov et al. (2004); in view of the relatively faint X-ray luminosities of the individual galaxies, the number of X-ray emitting galaxies required to account for the soft excess would be unrealistically high. 


\section{Soft X-Ray Emission Based on XMM-Newton EPIC Data}

\subsection{Continuum Detections}

\subsubsection{The Nevalainen et al. (2003) Sample}

Nevalainen et al. (2003) found evidence for soft X-ray excess in clusters of galaxies using XMM-Newton EPIC data. They derived a $20-40 \%$ soft excess in the central $500 \mathrm{kpc}$ regions of the clusters Coma, Abell 1795 and Abell $3112(z=0.0703)$ in channels below $2 \mathrm{keV}$, consistently in both PN and MOS instruments, and in ROSAT PSPC data.

A thermal model fits the data better than a non-thermal one, but at the level of calibration accuracy at the time, the non-thermal nature of the soft excess could not be ruled out.

Thermal modelling yielded temperatures in the range of $0.6-1.3 \mathrm{keV}$ and metal abundances consistent with zero. Assuming that this warm gas occupies the same volume as the hot gas, the electron densities are of the order of $10^{-4}-10^{-3} \mathrm{~cm}^{-3}$. These values lead to a cooling time scale larger than the Hubble time, i.e. the structures are self-consistent.

\subsubsection{The Kaastra et al. (2003) Sample}

Kaastra et al. (2003) examined a sample of 14 clusters of galaxies observed with XMMNewton, and found significant evidence for soft X-ray excess continuum emission in 5 of them: Coma (centre studied only, since it is closer than the other 4), Abell 1795, Sérsic 159-03, Abell 2052 and MKW 3s. Different modelling of the hot gas temperature and metal abundance yielded lower temperatures for the soft component $(\sim 0.2 \mathrm{keV})$, compared to Nevalainen et al. (2003). The surface brightness of the warm gas is rather constant with radius, while that of the hot gas decreases with radius, falling below the warm gas surface brightness between 0.5 and $1 \mathrm{Mpc}$ from the cluster centre. Note that in Coma, the central pointing does not cover this radius, so the hot gas brightness remains above the cool gas brightness, consistent with the above number. Such a behaviour is consistent with the Warm Hot Intergalactic Medium (hereafter WHIM) filament scenario, whereby the projected external filamentary structure is more extended than the cluster. Later, Kaastra et al. (2004) extended this sample to 21 clusters, and found 7 objects with soft excesses, the new cases being Abell 3112 (also in Nevalainen's paper) and Abell 2199.

Based on the quality of the spectral fits, in most cases the non-thermal model was also acceptable. Fitting the soft excess with a power-law model yielded rather constant photon indices $(\sim 2)$ with radius in a given cluster. At the cluster centre the luminosity of the nonthermal component is $\sim 10 \%$ of that of the hot gas, while the percentage increases towards $100 \%$ at the largest radii.

Bregman and Lloyd-Davies (2006) challenged the results of Kaastra et al. (2003), arguing that their soft excess detection was due to incorrect background subtraction. However, in a rebuttal paper, Nevalainen et al. (2007) showed that, especially in the central regions, the cluster emission is so bright compared to the background, that the details of the background modelling are insignificant. Thus, Bregman \& Lloyd-Davies's claim appears to be unjustified.

Nevalainen et al. (2007) also found that the changes in the EPIC calibration between the years 2002 and 2005 resulted in a decrease of the soft excess signal. Using the Kaastra et al. (2003) modelling, the PN soft excess even disappeared in some clusters. However, the MOS instrument still detects a soft excess in all the clusters of Kaastra's sample. A less conservative hot gas modelling (Nevalainen et al., in preparation) with the current calibration information obtains consistent soft excesses in both PN and MOS data. 


\subsubsection{Sérsic 159-03}

One of the first clusters to be observed by XMM-Newton was Sérsic 159-03. This observation with a net exposure time of $30 \mathrm{ks}$ was taken in 2000 (Kaastra et al. 2001). The same observation was used for a search for soft excess emission (Kaastra et al. 2003, see Sect. 4.1.2 above). A 60 ks observation taken two years later was analysed by Bonamente et al. (2005a) and de Plaa et al. (2006).

Bonamente et al. (2005a) confirmed the existence of the soft X-ray excess emission in Sérsic 159-03 out to a distance of $1 \mathrm{Mpc}$ from the cluster centre. The soft excess in the $0.3-1.0 \mathrm{keV}$ band increases from $10 \%$ at the centre to $80 \%$ at the largest radii. The properties of the soft excess differ from those derived for Sérsic 159-03 using PSPC data (Bonamente et al. 2001d), likely due to different modelling of the data: XMM-Newton allows to determine the hot gas component unambiguously in the $2.0-7.0 \mathrm{keV}$ band where the soft component has a negligible contribution.

The same dataset of Sérsic 159-03 was also analysed by de Plaa et al. (2006). However, they focused their attention on the chemical evolution of the cluster and they determined the background for the spectral analysis in the $9^{\prime}-12^{\prime}$ region around the cluster, subtracting the spatially extended soft excess emission together with the soft foreground emission. For the remaining soft excess in the cluster core they propose a non-thermal emission mechanism arising from IC scattering between $\mathrm{CMB}$ photons and relativistic electrons accelerated in bow shocks associated with ram pressure stripping of infalling galaxies.

Werner et al. (2007) studied Suzaku data together with the two XMM-Newton data sets of Sérsic 159-03 obtained two years apart mentioned before. They found consistent soft excess fluxes with all instruments in all observations. From the XMM-Newton data they derived radial profiles and $2 \mathrm{D}$ maps that show that the soft excess emission has a strong peak at the position of the central $\mathrm{cD}$ galaxy and does not show any significant azimuthal variations. They concluded that the spatial distribution of the soft excess is neither consistent with the models of intercluster warm-hot filaments, nor with models of clumpy warm intracluster gas associated with infalling groups as proposed by Bonamente et al. (2005a). Using the data obtained with the XMM-Newton RGS, Werner et al. could not confirm the presence of warm gas in the cluster centre with the expected properties assuming the soft excess was of thermal origin. They therefore concluded that the soft excess in Sérsic 159-03 is most probably of non-thermal origin.

\subsection{Line Emission Detection}

A crucial piece of evidence for the thermal nature of the soft X-ray excess would be the detection of emission lines. At the indicated temperatures of $0.1-0.5 \mathrm{keV}$ the most prominent emission lines are from $\mathrm{O}$ VII and $\mathrm{O}$ VIII. The resonance, intercombination and forbidden lines of O VII have energies of 574, 569 and $561 \mathrm{eV}$ (see also Kaastra et al. 2008-Chap. 9, this issue). For a low density plasma at $0.2 \mathrm{keV}$ temperature, the centroid of the triplet has an energy of $568.7 \mathrm{eV}$ (see Kaastra et al. 2003).

Geocoronal and heliospheric solar wind charge exchange (Wargelin et al. 2004; Fujimoto et al. 2007) also produces soft X-ray emission lines. These lines can vary by a factor of 3 on time scales of hours, which makes them difficult to model and subtract. The maximum observed brightness of these lines in the $0.5-0.9 \mathrm{keV}$ range can reach the level of the cosmic $\mathrm{X}$-ray background. Therefore, when the background level is important for the analysis, care should be taken when estimating the contaminating effects of this emission.

Finoguenov et al. (2003) observed a spatial variation of the soft excess emission in the Coma cluster. In the Coma 11 field they found an excess emission which is particularly 
strong. Recent observations with the Suzaku satellite (Takei et al., private communication) do not confirm the level of O VII and O VIII line emission reported by Finoguenov et al. (2003) for the Coma 11 field, but they are consistent with the lowest reported values for the other fields in Coma observed with XMM-Newton. They suggest that a large fraction of the reported excess soft X-ray emission and the line emission observed in the Coma 11 field with XMM-Newton was due to Solar wind charge exchange emission.

\subsubsection{The Kaastra et al. (2003) Sample}

Kaastra et al. (2003) found evidence for O VII line emission in the clusters Sérsic 159-03, MKW 3s $(z=0.0450)$ and Abell 2052 in form of line-like residuals on top of the hot gas model at wavelengths consistent with the cluster redshifts. Note that the line emission is significant only in the outer regions $\left(4^{\prime}-12^{\prime}\right)$ of the clusters.

The uncertainties also allow a Galactic origin $(z=0)$ for the emission. Also, Nevalainen et al. (2007) pointed out that a simple cluster-to-background flux level comparison does not exclude that the reported $\mathrm{O}$ VII line emission in the $0.5-0.65 \mathrm{keV}$ band contains contributions from the geocoronal and heliospheric Solar wind charge exchange (see above). This possibility remains to be studied in detail.

Therefore this study does not give conclusive evidence for O VII line emission in these three clusters.

\subsubsection{Coma}

Finoguenov et al. (2003) analysed XMM-Newton data in the outskirts of the Coma cluster. The spectrum at $\sim 1 \mathrm{Mpc}$ distance from the centre exhibits a very strong, $\sim 100 \%$ fractional soft excess continuum in channels below $0.8 \mathrm{keV}$, and the authors detect two emission lines in the $0.5-0.6 \mathrm{keV}$ band. Both the excess continuum and the line features are well fit with a thermal model of $\mathrm{k} T=0.22 \mathrm{keV}$ (see Fig. 3). The temperature, as well as the derived baryon overdensity for the warm gas $(\sim 200)$ are consistent with the WHIM filament properties in numerical simulations (e.g. Davé et al. 2001; Yoshikawa and Sasaki 2006). Let us note however that the soft excess in Finoguenov's model consists mostly of line emission, but due to the low spectral resolution of EPIC at low energy, it looks more like a continuum.

The redshift of the O VII line (0.0-0.026) is consistent with that of Coma (0.023) and also with being Galactic (0.0), implying that the emitter is located between us and Coma. Optical data show that there is a significant galaxy concentration in the direction of the warm gas in front of Coma. This suggests that the warm gas and the galaxy structure are connected, consistent with the WHIM filament scenario.

However, since the soft excess flux in Coma is comparable to the background level, the charge transfer flux in an active state (i.e. at the maximum observed level) can reach the soft excess level detected in Coma (Finoguenov et al. 2003). Bowyer and Vikhlinin (2004) use this coincidence as a proof that the soft excess detection in Coma is due to charge transfer. However, they did not model the soft excess spectrum with a charge transfer emission model to show that the model is consistent with the observed spectral features in Coma. Thus, their claim of Coma soft excess being due to charge transfer mechanism is not proven.

An indication for the presence of WHIM in Coma was also recently reported by Takei et al. (2007b) at $2.3 \sigma$ level in absorption and in emission, based on RGS spectra of the Active Galactic Nucleus X-Comae, which is located behind the Coma cluster. 
Fig. 3 Different emission components of the Coma spectrum obtained with the XMM-Newton PN instrument (from Finoguenov et al. 2003)

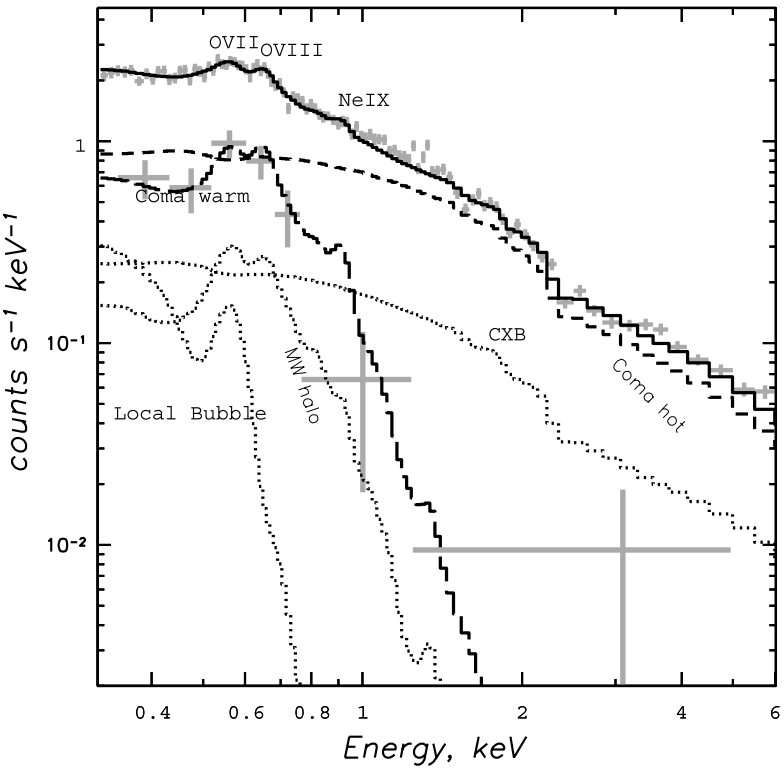

\section{Soft X-Ray Emission Based on Suzaku Observations}

\subsection{Search for the Soft Excess in and around Clusters}

Suzaku has observed about 30 clusters of galaxies by April 2007. Several of these observations were done in order to search for soft excess emission. We describe here the results for Abell 2218 and Abell 2052 in some detail since these two clusters have been studied rather extensively, and then briefly review results from other sources.

\subsection{Abell $2218(z=0.1756)$}

Abell 2218 is a bright cluster at $z=0.1756$ with an ICM temperature of about $7 \mathrm{keV}$. The Suzaku observations were carried out on two occasions with a total exposure of $80 \mathrm{ks}$ and the results were published by Takei et al. (2007a). Several conditions are favourable to the detection of a soft excess in this cluster with Suzaku. First, the cluster is known to be undergoing a merger, likely along the line of sight, as indicated by two galaxy concentrations at different redshifts. This picture is supported by X-ray observations from Chandra and by the presence of remarkable arcs due to gravitational lensing. Such a merger suggests that a large-scale filament may be located along the line of sight and that the column density of oxygen contained in the warm filament gas could be high. The second reason is that the XIS energy resolution allows to separate a $\sim 100 \mathrm{eV}$ cosmologically redshifted oxygen line from a zero redshift oxygen line. Background data in two regions at about $5^{\circ}$ offset from the cluster were also taken to obtain information on the oxygen line intensity of the Galactic component.

The time variation of the soft X-ray flux during the observations was very small, so the effect from the solar wind charge exchange emission was considered to be small. A soft excess was searched for in the outside region of the cluster, i.e. for the region with radius greater than $5^{\prime}$ (880 kpc at the source) from the Abell 2218 centre. The observed spectra 

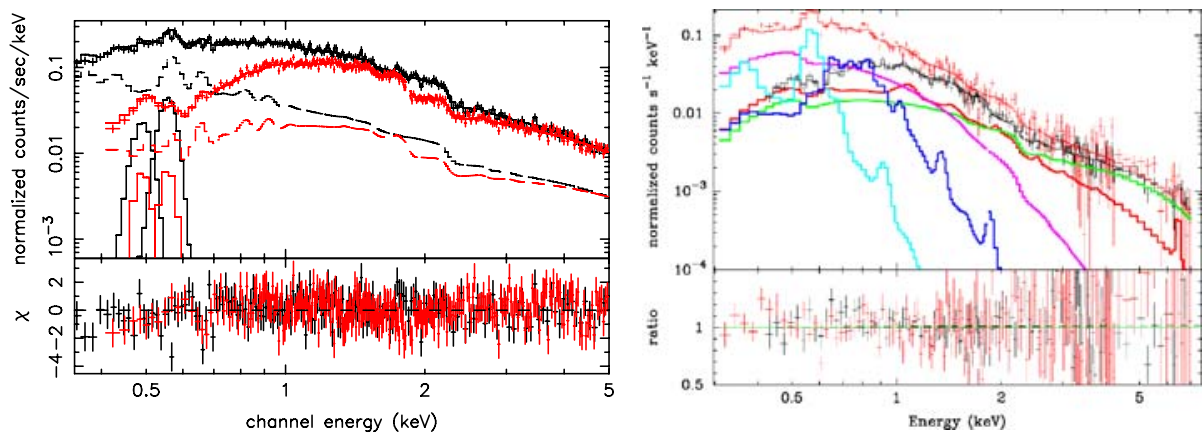

Fig. 4 Left: Observed XIS spectrum for the outer region of Abell 2218, with upper limits for the redshifted OVII and O VIII lines, for the BI (black) and FI (red) sensors. The background spectra are shown with dashed lines. This figure is taken from Takei et al. (2007a). Right: Observed XIS spectrum for $r=12^{\prime}-15^{\prime}$. Spectral fit including Galactic emission ( 0.1 and $0.3 \mathrm{keV}$ in light and dark blue), cosmic X-ray background (green), and thermal ICM emission with $\mathrm{k} T=2.8 \mathrm{keV}$ (red) requires an additional thermal Bremsstrahlung component with $\mathrm{k} T=0.7 \mathrm{keV}$ (magenta) whose intensity is constant over $r=0-20^{\prime}$. The summed up model (orange) is fitted to the data. Observations with Suzaku confirm the presence of soft excess emission in Sérsic 159-03 and its derived flux is consistent with the values determined with XMM-Newton (Sect. 4.1.3, Werner et al. 2007). However, Suzaku does not confirm the presence of the redshifted O VII lines in the cluster. The excess emission can be fit statistically equally well with a thermal model with low oxygen abundance $(<0.15$ Solar $)$ and with a non-thermal model. The figure is taken from Tamura et al. (2007)

(see Fig. 4) show structures around 0.5 and $0.6 \mathrm{keV}$, which are the energies corresponding to redshifted O VII and O VIII lines. However, close examination showed that the hump at $0.5 \mathrm{keV}$ can be caused by the oxygen edge in the XIS filters, and the peak around $0.6 \mathrm{keV}$ can be due to Galactic O VII line emission. This unfortunate situation hampered the original XIS capability of detecting the soft excess associated with the cluster. Takei et al. (2007a) set upper limits for the O VII and O VIII line intensities as shown in their Fig. 1, including all the uncertainties in the instrumental response (e.g. by the enhanced contamination in the XIS filters) and in the Galactic line intensities.

Even though the observing conditions were not optimal, the upper limits obtained are nearly an order of magnitude lower than the soft excess level reported in other clusters by Kaastra et al. (2003). With this upper limit, the density of the warm gas can be constrained. The limit for the gas density is:

$$
\delta \equiv n_{\mathrm{H}} / \bar{n}_{\mathrm{H}}<270\left(\frac{Z}{0.1 Z_{\odot}}\right)^{-1 / 2}\left(\frac{L}{2 \mathrm{Mpc}}\right)^{-1 / 2},
$$

where $\bar{n}_{\mathrm{H}}=X \Omega_{\mathrm{b}} \rho_{\text {crit }}(1+z)^{3} / m_{\mathrm{p}}=1.77 \times 10^{-7}(1+z)^{3} \mathrm{~cm}^{-3}$ is the mean hydrogen density in the universe, where $X=0.71$ is the hydrogen to total baryon mass ratio, $\Omega_{\mathrm{b}}=0.0457$ is the baryon density of the universe, $\rho_{\text {crit }}=9.21 \times 10^{-30} \mathrm{~g} \mathrm{~cm}^{-3}$ is the critical density of the universe, and $m_{\mathrm{p}}$ is the proton mass. Even though this level of gas density is much higher than the expected density $(\delta \sim 10)$ in the filaments, the result demonstrates that Suzaku can give a reasonable constraint on the soft excess emission.

\subsection{Abell 2052}

Suzaku carried out observations of Abell 2052 with 4 offset pointings to cover the cluster outskirts to about $20^{\prime}$ ( $830 \mathrm{kpc}$ at the source). Since the observations were all carried out only 
40 days after the launch, the contamination in the XIS filter was not so much a problem. At the carbon K-edge energy, the reduction of the transmission efficiency was less than $8 \%$ (see Koyama et al. 2007).

The data were analysed by Tamura et al. (2007). By assuming a single temperature for the ICM and two temperatures $(0.2$ and $0.6 \mathrm{keV})$ for the Galactic component, they found a significant soft excess, with a spectrum well described by a featureless continuum modelled (for convenience reasons only) by pure thermal Bremsstrahlung with a temperature around $0.7 \mathrm{keV}$. The intensity of this soft component is consistent with a constant value over the entire observed field and stronger than the Galactic emission below $0.5 \mathrm{keV}$. If this emission is associated with Abell 2052, then its luminosity can be comparable to the ICM emission which shows $L_{X} \sim 1.4 \times 10^{44} \mathrm{erg} \mathrm{s}^{-1}$ in the $2-10 \mathrm{keV}$ band. The cluster is located fairly close to the extension of the North Polar Spur (NPS), a large spur-like region with strong soft X-ray emission (see e.g. Willingale et al. 2003). However, the Suzaku spectrum of the NPS region showed the temperature to be about $0.3 \mathrm{keV}$ with a fairly strong $\mathrm{Mg}-\mathrm{K}$ emission line. This is inconsistent with the Abell 2052 soft excess spectrum.

A strong soft component without emission lines may be caused by extended non-thermal emission. Since the cluster shows neither strong radio emission nor merger features, such a non-thermal emission could be due to a rather old population of non-thermal electrons (see e.g. Rephaeli et al. 2008-Chap. 5, this issue).

\subsection{Other Clusters}

The Sculptor supercluster was observed with Suzaku in 4 pointings with the fields partially overlapping and connecting 3 main clusters (Abell 2811 at $z=0.1086$, Abell 2804 at $z=$ 0.11245 and Abell 2801 at $z=0.11259$ ). The data were analysed by Kelley et al. (2007). The combined spectrum after removing bright clusters and point sources shows an excess in the energy range $0.6-1 \mathrm{keV}$, with a temperature of about $0.8 \mathrm{keV}$. Since the observed volume is extremely large $\left(\sim 500 \mathrm{Mpc}^{3}\right)$, the uniform electron density implied is as low as $8 \times 10^{-6} \mathrm{~cm}^{-3}$.

The Suzaku XIS data on Abell 1060 ( $z=0.01140$, Sato et al. 2007), AWM7 ( $z=$ 0.01724, Sato et al. 2008), and Fornax (Matsushita et al. 2007) have been analysed in some detail. Even though the spectrum and intensity of the foreground Galactic emission have fairly large ambiguities, the observed energy spectra for these clusters are generally well fit by thermal models at energies down to about $0.3 \mathrm{keV}$. For the central region of Fornax $\left(r<2^{\prime}\right)$, a two temperature model with $\mathrm{k} T=1.5$ and $0.8 \mathrm{keV}$ was preferred. However, excess emission which causes significant deviation from these standard spectral fits has not been detected from these clusters.

Fujita et al. (2007) observed the region between Abell 399 and Abell 401 with Suzaku. They found no evidence for oxygen emission from the WHIM in this region and obtained a strict upper limit of $4.1 \times 10^{-5} \mathrm{~cm}^{-3}$ on its density.

\section{Soft X-Ray Emission Based on Chandra Observations}

Reports on soft excess emission in clusters as observed by Chandra are scarce. Henriksen et al. (2004) detected soft excess emission in the merging cluster Abell $754(z=0.0535)$. Although a non-thermal origin could not be fully excluded, from their combined Chandra, BeppoSAX, ASCA and ROSAT PSPC analysis they conclude that a weak soft thermal component $(\sim 1 \mathrm{keV})$ is present in this hot $(\sim 10 \mathrm{keV})$ cluster. The emission extends out to $8^{\prime}$ 
from the core and is peaked in the cluster centre. Henriksen et al. attribute the emission to embedded groups of galaxies.

In another cluster, Abell $2163(z=0.2030)$, Henriksen and Hudson (2004) also report a soft and hard X-ray excess in their combined Chandra and ROSAT PSPC analysis. The excess can be modelled by a non-thermal component with photon index 2.7-5.9. Little detail about the observations is given in this paper, but the authors attribute the non-thermal emission to a weak merger shock that is present in this cluster.

Bonamente et al. (2007) analysed Chandra data of Abell 3112 in the central 1.0-2.5 arcmin region and found significant soft excess, reaching $20 \%$ of the hot gas emission level at $0.3 \mathrm{keV}$. Its spectrum is acceptably fit with a low temperature $(\sim 0.5 \mathrm{keV})$ thermal model or by a non-thermal model $\left(\alpha_{\mathrm{ph}} \sim 1.8\right)$. The best-fit values differ from those obtained with XMM-Newton data, indicating that remaining calibration uncertainties affect fine details. Nevertheless, the magnitude of the soft excess is above the level of calibration inaccuracies, and similar to that in XMM-Newton, thus proving the celestial origin of the soft excess in Abell 3112.

\section{Discussion}

Soft excess emission, defined here as excess emission over the usual thermal emission from hot intracluster gas at energies below $1 \mathrm{keV}$, has been detected significantly in $\sim 30 \%$ of clusters of galaxies. This value is based on the two largest cluster soft excess samples (Bonamente et al. 2003; Kaastra et al. 2003). However, as described above, such detections are difficult because of instrumental issues and for many objects a controversy remains.

Thermal and non-thermal models have been proposed to account for this soft excess emission. We will briefly summarise them below using Coma and Sérsic 159-03 as examples.

\subsection{Thermal Models}

\subsubsection{WHIM in Coma}

The massive soft excess halo around Coma (Bonamente et al. 2003) is not well accounted for by a non-thermal model. Furthermore, Finoguenov et al. (2003) detected O VII and O VIII line emission in the outskirts of Coma. Thus Coma is a good case for a cluster where the soft excess is dominantly of thermal nature. Bonamente et al. (2003) proposed that the soft component may reside in external filamentary structures of warm-hot intergalactic medium (WHIM). These filaments are predicted by hydrodynamic simulations of formation and evolution of large-scale structures, extending over several megaparsecs, containing a large fraction of the current epoch's baryons (e.g. Cen and Ostriker 1999; Davé et al. 2001; Yoshikawa and Sasaki 2006). In WHIM simulations, the temperatures are in the range $10^{5}$ $10^{7} \mathrm{~K}$, consistent with the soft excess properties found in Coma. Assuming a filamentary geometry, Bonamente et al. (2003) derived densities of $n \sim 10^{-5}-10^{-4} \mathrm{~cm}^{-3}$ for the warm gas in Coma. These values are consistent with those in the WHIM simulations $\left(10^{-7}\right.$ $10^{-4} \mathrm{~cm}^{-3}$ ). Assuming $n=10^{-4} \mathrm{~cm}^{-3}$, the implied mass of the filament exceeds that of the hot gas by a factor of 3 . 


\subsubsection{Soft Emission from Merging Sub-halos}

In Sérsic 159-03, detailed WHIM filament calculations showed that unrealistically long structures $(\sim 100-1000 \mathrm{Mpc})$, projected on the line of sight of the cluster, are required to explain the soft excess detected in XMM-Newton data (Bonamente et al. 2005a). The assumption that the warm gas occupies the same volume as the hot gas has the problem that there is a pressure difference between the two components, and that the cooling time is short. As proposed by these authors, this problem can be avoided if one assumes that the warm gas is not distributed evenly but rather in high density $10^{-2}-10^{-3} \mathrm{~cm}^{-3}$ clumps with a volume filling factor $f \ll 1$. Such a distribution is predicted by the simulations of Cheng et al. (2005), in which the soft excess emission comes from high-density and low entropy gas associated with merging sub-groups, which preserve their identity before being destroyed and thermalised in the hot ICM. The simulated $0.2-1.0 \mathrm{keV}$ band soft excess in the radial range of $0-0.5 r_{\text {vir }}$ is consistent with that published in Bonamente et al. (2005a) for Sérsic 159-03. This scenario yields a warm gas mass of $25 \%$ of the hot gas mass for Sérsic 159-03.

However, Werner et al. (2007) show that the soft excess emission peaks at the position of the central $\mathrm{cD}$ galaxy and does not show any significant azimuthal variations. Moreover the soft excess in Sérsic 159-03 is observed out to radii of at least $1 \mathrm{Mpc}$. If this soft excess is associated with the gas of an infalling group, then this group is moving exactly along the line of sight. However, such an infalling group cannot explain the presence of the soft excess emission at large radii. Therefore the soft excess observed in Sérsic 159-03 is most probably not of thermal origin.

\subsection{Non-thermal Models}

Inverse Compton models of energetic electrons on CMB photons and/or on galaxy starlight have been developed by various authors to account for the soft excess observed in several of the objects presented here.

Non-thermal inverse Compton emission has a power-law spectrum with a relative flux which in the $0.3-10.0 \mathrm{keV}$ band may account for more than $30 \%$ of the cluster emission. The best-fit power-law photon indices of soft excess clusters are typically between $\alpha_{\mathrm{ph}} \sim 2.0-2.5$. In the IC model, this corresponds to a differential relativistic electron number distribution $\mathrm{d} N / \mathrm{d} E=N_{0} E^{(-\mu)}$ with $\mu=3-4$. These are steeper than the distribution of the Galactic cosmic-ray electrons $(\mu \sim 2.7)$. The steeper power-law distribution might indicate that the relativistic electrons suffered radiative losses (Sarazin 1999). Relativistic electrons in this energy range have relatively long lifetimes of $t_{\mathrm{IC}}=2.3 \times 10^{9}\left(\gamma / 10^{3}\right)^{-1}(1+z)^{-4} \mathrm{yr}$ and $t_{\text {syn }}=2.4 \times 10^{10}\left(\gamma / 10^{3}\right)^{-1}(B /(1 \mu G))^{-2} \mathrm{yr}$ for inverse-Compton and synchrotron processes respectively.

As mentioned earlier, Werner et al. (2007) conclude that a non-thermal model best explains the observed properties of the soft excess in Sérsic 159-03. The total energy in relativistic electrons needed to explain the excess emission within the radius of $600 \mathrm{kpc}$ does not exceed $1 \times 10^{61} \mathrm{erg}$, while the total thermal energy within the same radius is $3 \times 10^{63} \mathrm{erg}$. This means that even if the energy in relativistic ions is as much as $\sim 30$ times larger than that in relativistic electrons, the total energy in cosmic ray particles will only account for $10 \%$ of the thermal energy of the ICM.

Models that may account for the observed soft excess can also be found in the following list: Enßlin et al. (1999); Atoyan and Völk (2000); Sarazin and Kempner (2000); Takizawa and Naito (2000); Fujita and Sarazin (2001); Petrosian (2001); De Paolis et al. (2003); Bowyer et al. (2004); Petrosian et al. (2008)—Chap. 10, this issue. 


\subsection{Some Problems and Open Questions}

In the Virgo cluster a strong soft excess was detected in the extreme ultraviolet with EUVE (Lieu et al. 1996a; Berghöfer et al. 2000a; Bonamente et al. 2001b; Durret et al. 2002) and in the 0.2-0.4 keV band with ROSAT (Bonamente et al. 2002). However, the observations with XMM-Newton did not confirm the existence of this soft excess: a thermal model for the hot cluster emission with Galactic absorption describes the soft band X-ray spectra (above $0.3 \mathrm{keV}$ ) of Virgo obtained with XMM-Newton sufficiently well (Kaastra et al. 2003; Matsushita et al. 2002).

Arabadjis and Bregman (1999) claimed that some of the X-ray absorption cross sections were wrong, and that soft excesses would disappear when using the proper values. However, it is surprising that this claim has neither been confirmed nor refuted ever since. During some time, there have been wrong He cross sections by Balucinska-Church and McCammon (1992) in the XSPEC software (see http://heasarc.gsfc.nasa.gov/docs/xanadu/xspec/). Arabadjis \& Bregman refer to those wrong cross sections. They have been improved by Yan et al. (1998) and are now included properly for example in Wilms' cross sections that are in XSPEC. They are also properly included in the SPEX software. Note that Wilms' more recent cross sections agree quite well with the older Morrison and McCammon (1983) work (see the discussion in Wilms et al. 2000). Lesson to be learned: it is important to check for each paper which cross sections/absorption model have been used!

Several searches for far-ultraviolet emission lines expected from a $10^{6} \mathrm{~K}$ gas were performed with the FUSE satellite on the cores of several clusters. Oegerle et al. (2001) have reported the detection of O VI $\lambda=1032 \AA$ in Abell $2597(z=0.0824)$, implying a mass inflow rate of about $40 \mathrm{M}_{\odot} / \mathrm{yr}$. However, FUSE has not detected warm gas in five other clusters: Abell 1795 (Oegerle et al. 2001), Coma and Virgo (Dixon et al. 2001), and Abell 2029 $(z=0.0775)$ and Abell 3112 (Lecavelier des Etangs et al. 2004); the upper limits on the inflow rates for these five clusters were of the order $25 \mathrm{M}_{\odot} / \mathrm{yr}$.

A recent review by Bregman (2007) summarises current knowledge on the search for missing baryons at low redshift. Note however that in his Fig. 9 (already in Bregman and Lloyd-Davies 2006) he claims that the soft excess found in some clusters by Kaastra et al. (2003) is an artefact due to wrong background subtraction. The argument is that there is a correlation between the Rosat R12 (low energy) count rate and the presence or absence of a soft excess. However, a closer inspection shows that this correlation is driven by a few clusters with strong Galactic absorption. As Kaastra et al. (2003) pointed out, in addition to the atomic gas visible at $21 \mathrm{~cm}$ these clusters also have significant X-ray opacity contributions due to dust or molecules, which naturally explains the observed flux deficit. When these clusters are excluded, the correlation fades away.

\section{Note Added in Proof}

Until very recently, the Galactic neutral hydrogen absorption taken into account when fitting X-ray spectra was extracted from the Dickey and Lockman (1990, hereafter DL90) survey. A better all-sky survey, the Leiden/Argentine/Bonn (LAB) Galactic H I Survey, has now become available (Kalberla et al. 2005). Though its use is not yet worldwide, it has been pointed out that, at least in some cases, the LAB survey gives notably smaller values than DL90 for the H I absorption. For example, in the case of Abell 3112, the LAB value is $1.3 \times$ $10^{20} \mathrm{~cm}^{-2}$ while the DL90 value is $2.6 \times 10^{20} \mathrm{~cm}^{-2}$, and for Sérsic 159-03 (= AS 1101), the LAB value is $1.14 \times 10^{20} \mathrm{~cm}^{-2}$, while the DL90 value is $1.79 \times 10^{20} \mathrm{~cm}^{-2}$. Such changes 
Fig. 5 XMM-Newton MOS data of Abell 3112 in a 1.5-2.9 arcmin annulus and fit with a single temperature MEKAL model in the $2-7 \mathrm{keV}$ band with the LAB $\mathrm{H}$ I absorption column, showing a $20 \%$ soft excess

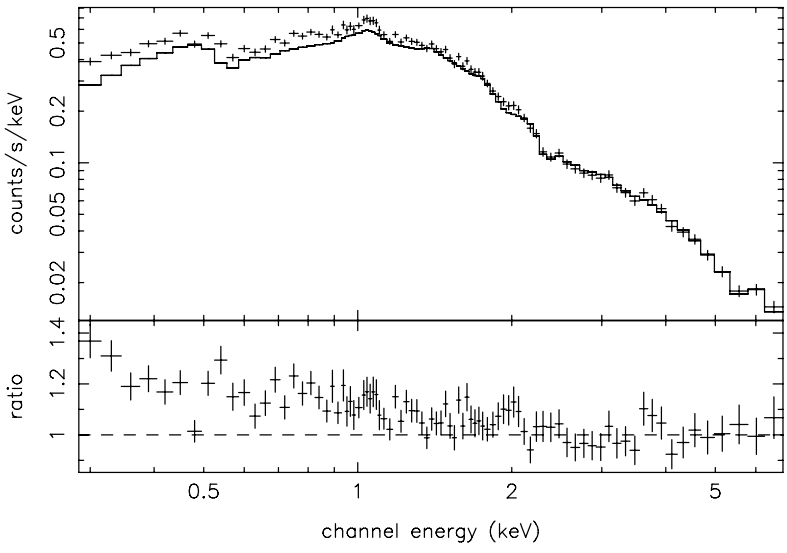

are expected to modify the soft excess emission derived from spectral fits. Fortunately, for many other clusters the differences between the $\mathrm{H}$ I absorption measured by LAB and by DL90 are negligible.

For Abell 3112, a fit of the XMM-Newton MOS data in a 1.5-2.9 arcmin annulus made by J. Nevalainen with a single temperature MEKAL model in the 2-7 keV band and extrapolated down to $0.3 \mathrm{keV}$ using the LAB H I absorption value shows that a $20 \%$ soft excess remains (see Fig. 5). In order to eliminate the soft excess, the H I absorption should take the unrealistically low value of $1.510^{19} \mathrm{~cm}^{-2}$.

For Sérsic 159-03, new fits of the joint Suzaku and XMM-Newton data in a 3-8 arcmin annulus were made by $\mathrm{N}$. Werner using the new $\mathrm{H}$ I LAB absorption value. If the absorption is a free parameter in the fit, $\mathrm{N}_{\mathrm{H}}$ goes to zero, and no good fit can be obtained with $N_{\mathrm{H}}=$ $1.14 \times 10^{20} \mathrm{~cm}^{-2}$. Adding a thermal component with a temperature of $0.2 \mathrm{keV}$ improves the fit significantly (from a reduced $\chi^{2}$ of 2.8 to 1.5), and the soft component is found to be present at a significance level of $13.5 \sigma$. If the fit is made with a multi-temperature model that accounts for the cluster emission with the absorption left as a free parameter, a $2 \sigma$ upper limit for $N_{\mathrm{H}}$ of $1.4 \times 10^{19} \mathrm{~cm}^{-2}$ is obtained; this value is inconsistent with the new LAB survey and clearly unphysically low. In the same way, if a power law is fit to the data, even if its total flux is reduced by about $20 \%$ it remains significant at a $\sim 10 \sigma$ level. A soft component is therefore required even for zero absorption, implying that the soft excess is really strong in this cluster.

Therefore, even if they are somewhat reduced, the soft excesses observed in Abell 3112 and Sérsic 159-03 remain when taking into account the new values of the $\mathrm{H}$ I absorption.

\section{Conclusions}

While in most cases the origin of the soft excess emission is difficult to prove unambiguously, due to problems with instrument calibration and unknown background/foreground emission level, the independent detection of the phenomenon with many instruments gives confidence in the genuine nature of the phenomenon in a number of clusters. Both thermal and non-thermal emission mechanisms are probably at work in producing the soft excess emission in clusters. 
Acknowledgements The authors thank ISSI (Bern) for support of the team "Non-virialized X-ray components in clusters of galaxies". F.D. acknowledges support from CNES. SRON is supported financially by NWO, the Netherlands Foundation for Scientific Research. J.N. acknowledges support from the Academy of Finland. We thank R. Lieu, M. Bonamente and A. Fabian for pointing out the possible modifications of the soft excess emission when using more recent determinations of the hydrogen absorption along the line of sight (see note added in proof).

\section{References}

J.S. Arabadjis, J.N. Bregman, Astrophys. J. 514, 607 (1999)

A.M. Atoyan, H.J. Völk, Astrophys. J. 535, 45 (2000)

M. Balucińska-Church, D. McCammon, Astrophys. J. 400, 699 (1992)

T.W. Berghöfer, S. Bowyer, Astrophys. J. 565, L17 (2002)

T.W. Berghöfer, S. Bowyer, E. Korpela, Astrophys. J. 535, 615 (2000a)

T.W. Berghöfer, S. Bowyer, E. Korpela, Astrophys. J. 545, 695 (2000b)

M. Bonamente, R. Lieu, J.P.D. Mittaz, Astrophys. J. 546, 805 (2001a)

M. Bonamente, R. Lieu, J.P.D. Mittaz, Astrophys. J. 547, L7 (2001b)

M. Bonamente, R. Lieu, J. Nevalainen, J.S. Kaastra, Astrophys. J. 552, L7 (2001c)

M. Bonamente, R. Lieu, J.P.D. Mittaz, Astrophys. J. 561, L63 (2001d)

M. Bonamente, R. Lieu, M.K. Joy, J.H. Nevalainen, Astrophys. J. 576, L688 (2002)

M. Bonamente, M.K. Joy, R. Lieu, Astrophys. J. 585, 722 (2003)

M. Bonamente, R. Lieu, J. Mittaz, J.S. Kaastra, J. Nevalainen, Astrophys. J. 629, 192 (2005a)

M. Bonamente, R. Lieu, J. Kaastra, Astron. Astrophys. 443, 29 (2005b)

M. Bonamente, J. Nevalainen, R. Lieu, Astrophys. J. 668, 796 (2007)

S. Bowyer, T.W. Berghöfer, Astrophys. J. 506, 502 (1998)

S. Bowyer, R.F. Malina, in Extreme Ultraviolet Astronomy, ed. by R.F. Malina, S. Bowyer (Pergamon, New York, 1991), p. 397

S. Bowyer, A. Vikhlinin, J. Korean Astron. Soc. 37, 579 (2004)

S. Bowyer, T.W. Berghöfer, E.J. Korpela, Astrophys. J. 526, 592 (1999)

S. Bowyer, E.J. Korpela, T.W. Berghöfer, Astrophys. J. 548, L35 (2001)

S. Bowyer, E.J. Korpela, M. Lampton, T.W. Jones, Astrophys. J. 605, 168 (2004)

J.N. Bregman, Annu. Rev. Astron. Astrophys. 45, 221 (2007)

J. Bregman, E.J. Lloyd-Davies, Astrophys. J. 644, 167 (2006)

U. Briel, B. Aschenbach, G. Hasinger et al., http://heasarc.gsfc.nasa.gov/docs/rosat/ruh/handbook/ rosathandbook.html (1996)

R. Cen, J.P. Ostriker, Astrophys. J. 514, 1 (1999)

L.-M. Cheng, S. Borgani, P. Tozzi et al., Astron. Astrophys. 431, 405 (2005)

R. Davé, R. Cen, J.P. Ostriker et al., Astrophys. J. 552, 473 (2001)

F. De Paolis, G. Ingrosso, A.A. Nucita, D. Orlando, Astron. Astrophys. 398, 435 (2003)

J. de Plaa, N. Werner, A.M. Bykov et al., Astron. Astrophys. 452, 397 (2006)

J.M. Dickey, F.J. Lockman, Annu. Rev. Astron. Astrophys. 28, 215 (1990)

W.V.D. Dixon, S. Sallmen, M. Hurwitz, R. Lieu, Astrophys. J. 550, L25 (2001)

F. Durret, E. Slezak, R. Lieu, S. Dos Santos, M. Bonamente, Astron. Astrophys. 390, 397 (2002)

M. Ehle, R. Breitfellner, R. González-Riestra et al., http://xmm.vilspa.esa.es/external/xmm_user_support/ documentation/uhb/index.html (2006)

T.A. Enßlin, R. Lieu, P.L. Biermann, Astron. Astrophys. 344, 409 (1999)

A. Finoguenov, U.G. Briel, J.P. Henry, Astron. Astrophys. 410, 777 (2003)

A. Finoguenov, U.G. Briel, J.P. Henry et al., Astron. Astrophys. 419, 47 (2004)

Y. Fujita, C.L. Sarazin, Astrophys. J. 563, 660 (2001)

Y. Fujita, N. Tawa, K. Hayashida et al., Publ. Astron. Soc. Jpn. (2007 in press) (arXiv:0705.2017)

R. Fujimoto, K. Mitsuda, D. McCammon et al., Publ. Astron. Soc. Jpn. 59, S133 (2007)

M.J. Henriksen, D.S. Hudson, J. Korean Astron. Soc. 37, 299 (2004)

M.J. Henriksen, D.S. Hudson, E. Tittley, Astrophys. J. 610, 762 (2004)

J.S. Kaastra, R. Lieu, J.P.D. Mittaz et al., Astrophys. J. 519, L119 (1999)

J.S. Kaastra, C. Ferrigno, T. Tamura et al., Astron. Astrophys. 365, L99 (2001)

J.S. Kaastra, R. Lieu, J.A.M. Bleeker, R. Mewe, S. Colafrancesco, Astrophys. J. 574, L1 (2002)

J.S. Kaastra, R. Lieu, T. Tamura, F.B.S. Paerels, J. den Herder, Astron. Astrophys. 397, 445 (2003)

J.S. Kaastra, R. Lieu, T. Tamura, F.B.S. Paerels, J.W.A. den Herder, in Soft X-Ray Emission from Clusters of Galaxies and Related Phenomena, ed. by R. Lieu, J. Mittaz, Astrophysics \& Space Science Library, vol. 309 (2004), p. 37 
J.S. Kaastra, F. Paerels, F. Durret, S. Schindler, P. Richter, Space Sci. Rev. (2008). doi:10.1007/s11214-008-9310-4

P.M.W. Kalberla, W.B. Burton, D. Hartmann et al., Astron. Astrophys. 440, 775 (2005)

R.L. Kelley, K. Mitsuda, C.A. Allen et al., Publ. Astron. Soc. Jpn. 59, S7 (2007)

K. Koyama, H. Tsunemi, T. Dotani et al., Publ. Astron. Soc. Jpn. 59, S23 (2007)

A. Lecavelier des Etangs, Gopal-Krishna, F. Durret, Astron. Astrophys. 421, 503 (2004)

R. Lieu, J.P.D. Mittaz, S. Bowyer et al., Astrophys. J. 458, L5 (1996a)

R. Lieu, J.P.D. Mittaz, S. Bowyer et al., Science 274, 1335 (1996b)

R. Lieu, W.-H. Ip, W.I. Axford, M. Bonamente, Astrophys. J. 510, L25 (1999a)

R. Lieu, M. Bonamente, J.P.D. Mittaz, Astrophys. J. 517, L91 (1999b)

R. Lieu, M. Bonamente, J.P.D. Mittaz et al., Astrophys. J. 527, L77 (1999c)

R. Lieu, M. Bonamente, J.P.D. Mittaz et al., Astron. Astrophys. 364, 497 (2000)

K. Matsushita, E. Belsole, A. Finoguenov, H. Böhringer, Astron. Astrophys. 386, 77 (2002)

K. Matsushita, Y. Fukazawa, J.P. Hughes et al., Publ. Astron. Soc. Jpn. 59, S327 (2007)

K. Mitsuda, M. Bautz, H. Inoue et al., Publ. Astron. Soc. Jpn. 59, S1 (2007)

J.P.D. Mittaz, R. Lieu, F.J. Lockman, Astrophys. J. 498, L17 (1998)

R. Morrison, D. McCammon, Astrophys. J. 270, 119 (1983)

J. Nevalainen, R. Lieu, M. Bonamente, D. Lumb, Astrophys. J. 584, 716 (2003)

J. Nevalainen, M. Bonamente, J. Kaastra, Astrophys. J. 656, 733 (2007)

W.R. Oegerle, L. Cowie, A. Davidsen et al., Astrophys. J. 560, 187 (2001)

V. Petrosian, Astrophys. J. 557, 560 (2001)

V. Petrosian, Y. Rephaeli, A.M. Bykov, Space Sci. Rev. (2008). doi:10.1007/s11214-008-9327-2

Y. Rephaeli, J. Nevalainen, T. Ohashi, A. Bykov, Space Sci. Rev. (2008). doi:10.1007/s11214-008-9314-7

C.L. Sarazin, Astrophys. J. 520, 529 (1999)

C.L. Sarazin, J.C. Kempner, Astrophys. J. 533, 73 (2000)

C.L. Sarazin, R. Lieu, Astrophys. J. 494, L177 (1998)

K. Sato, N.Y. Yamasaki, M. Ishida et al., Publ. Astron. Soc. Jpn. 59, 299 (2007)

K. Sato, K. Matsushita, Y. Ishisaki, N.Y. Yamasaki, M. Ishida, S. Sasaki, T. Ohashi, Publ. Astron. Soc. Jpn. (2008 in press). arXiv:0707.4342

P.J. Serlemitsos, Y. Soong, K.-W. Chan et al., Publ. Astron. Soc. Jpn. 59, S9 (2007)

L. Strüder, U. Briel, K. Dennerl et al., Astron. Astrophys. 365, L18 (2001)

Y. Takei, J.P. Henry, A. Finoguenov et al., Publ. Astron. Soc. Jpn. 59, S339 (2007a)

Y. Takei, J.P. Henry, A. Finoguenov et al., Astrophys. J. 655, 831 (2007b)

M. Takizawa, T. Naito, Astrophys. J. 535, 586 (2000)

T. Tamura, Y. Takei, K. Mitsuda, T. Ohashi, K. Matsushita, the Suzaku A 2052 team, Proc. "Tracing Cosmic Evolution with Clusters of Galaxies: Six Years Later”, Sesto, Italy, 25-29 June 2007

M.J.L. Turner, A. Abbey, M. Arnaud et al., Astron. Astrophys. 365, L27 (2001)

B.J. Wargelin, M. Markevitch, M. Juda et al., Astrophys. J. 607, 596 (2004)

N. Werner, J.S. Kaastra, Y. Takei et al., Astron. Astrophys. 468, 849 (2007)

R. Willingale, A.D.P. Hands, R.S. Warwick, S.L. Snowden, D.N. Burrows, Mon. Not. R. Astron. Soc. 343, 995 (2003)

J. Wilms, A. Allen, R. McCray, Astrophys. J. 542, 914 (2000)

M. Yan, H.R. Sadeghpour, A. Dalgarno, Astrophys. J. 496, 1044 (1998)

K. Yoshikawa, S. Sasaki, Publ. Astron. Soc. Jpn. 58, 641 (2006) 